\title{
A Jacobi-Collocation Method for Second Kind Volterra Integral Equations with a Smooth Kernel
}

\author{
Hongfeng Guo, Haotao Cai, and Xin Zhang \\ School of Mathematics and Quantitative Economics, Shandong University of Finance and Economics, Jinan, Shandong 250014, China \\ Correspondence should be addressed to Xin Zhang; zhangxin80@sdufe.edu.cn
}

Received 28 May 2014; Revised 2 July 2014; Accepted 2 July 2014; Published 17 July 2014

Academic Editor: Juan J. Nieto

Copyright (c) 2014 Hongfeng Guo et al. This is an open access article distributed under the Creative Commons Attribution License, which permits unrestricted use, distribution, and reproduction in any medium, provided the original work is properly cited.

\begin{abstract}
The purpose of this paper is to provide a Jacobi-collocation method for solving second kind Volterra integral equations with a smooth kernel. This method leads to a fully discrete integral operator. First, it is shown that the fully discrete integral operator is stable in both $L^{\infty}$ and weighted $L^{2}$ norms. Then, the proposed approach is proved to arrive at an optimal (the most possible) convergent order in both norms. One numerical example demonstrates the efficiency and accuracy of the proposed method.
\end{abstract}

\section{Introduction}

In this paper, we provide a Jacobi-collocation approach for solving the second kind Volterra integral equation of the form

$$
u(x)+\int_{-1}^{x} k(x, t) u(t) d t=f(x), \quad x \in I:=[-1,1]
$$

where the kernel function $k$ and the input function $f$ are given smooth functions about their variables and $u$ is the unknown function to be determined.

For ease of analysis, we will write (1) into an operator form. By introducing the integral operator $\mathscr{K}$ by

$$
(\mathscr{K} v)(x):=\int_{-1}^{x} k(x, t) v(t) d t, \quad x \in I,
$$

(1) is reformulated as

$$
(\mathscr{I}+\mathscr{K}) u=f .
$$

It is well known that there are many numerical methods for solving second kind Volterra integral equations such as the Runge-Kutta method and the collocation method based on piecewise polynomials; see, for example, Brunner [1] and references therein. For more information of the progress on the study of the problem, we refer the readers to [2-8]. Recently, a few works touched the spectral approximation to Volterra integral equations. In [9], Elnagar and Kazemi provided a novel Chebyshev spectral method for solving nonlinear Volterra-Hammerstein integral equations. Then, this method was investigated by Fujiwara in [10] for solving the first kind Fredholm integral equation under multipleprecision arithmetic. Nevertheless, no theoretical results were provided to justify the high accuracy. In [11], Tang et al. developed a novel Legendre-collocation method for solving (3). Inspired by the work of [11], Chen and Tang in $[5,12]$ obtained the spectral Jacobi-collocation method for solving the second kind Volterra integral equations with general weakly singular kernels $k(x, t)(x-t)^{-\mu}$ for $-1<\mu<0$. In [13], a spectral and pseudospectral Jacobi-Galerkin approach was presented for solving (3). In [14], Wei and Chen considered a spectral Jacobi-collocation method for solving Volterra type integrodifferential equation. In [15], Cai considered a Jacobicollocation method for solving Fredholm integral equations of second kind with weakly singular kernels.

Unfortunately, all these papers [5, 11-14] give the convergence analysis but suffer from the stability analysis. Because of lack of the stability analysis, the approximate solutiondoes not attain the most possible convergence order. Moreover, all of those papers do not answer that the approximate equation has a unique solution. Hence, in this paper, we will provide a Jacobi-collocation method for solving (3), which extends the Legendre spectral method developed in [11]. This spectral method leads to a fully discrete linear system. We are going to show that the fully discrete integral operator is stabile; that 
is, the approximate equation has a unique solution, and then, present the optimal (the most possible) convergent order of the approximate solution based on the stability analysis. We organize this paper as follows. In Section 2, as demonstrated in [13], we review a spectral Jacobi-collocation method for solving (3). In Section 3, a few important results are presented to analyze the Jacobi-collocation approach. In Sections 4 and 5, we analyze the Jacobi-collocation method, including the stability of the approximate equation and the convergent order of the approximate solution, in both $L^{\infty}$ and weighted $L^{2}$ norms, respectively. In Section 6, one numerical example is presented to show the efficiency and accuracy of this method.

The problem under study deserves more investigations in future works. Moreover, we believe that the semianalytical approaches are useful to investigate the problem. For related terminologies and applications of semianalytical approaches, please refer to [16-18].

\section{A Spectral Jacobi-Collocation Method}

In this section, we are going to review the spectral Jacobicollocation method for solving (3). To this end, we introduce several index sets: $\mathbb{N}:=\{1,2, \ldots, n, \ldots\}, \mathbb{N}_{0}:=\mathbb{N} \cup\{0\}$ and $\mathbb{Z}_{n}:=\{0,1,2, \ldots, n\}$. We let $w^{\alpha, \beta}(x):=(1-x)^{\alpha}(1+x)^{\beta}$ for $\alpha, \beta>-1$ be a weight function and then use the notation $L_{w^{\alpha, \beta}}^{2}(I)$ to be the set of all square integrable functions associated with the weight function $w^{\alpha, \beta}$, equipped with the norm

$$
\|v\|_{w^{\alpha, \beta}}:=\left(\int_{I} w^{\alpha, \beta}(t) v^{2}(t) d t\right)^{1 / 2}
$$

For $n \in \mathbb{N}$, we denote the points by $x_{i}^{\alpha, \beta}, i \in \mathbb{Z}_{n}$ to be the set of $n+1$ Jacobi-Gauss points corresponding to the Jacobi weight function $w^{\alpha, \beta}$. By introducing

$$
\pi(x):=\left(x-x_{0}^{\alpha, \beta}\right)\left(x-x_{1}^{\alpha, \beta}\right) \cdots\left(x-x_{n}^{\alpha, \beta}\right),
$$

we define the Lagrange fundamental interpolation polyno$\operatorname{mial} L_{i}^{\alpha, \beta}, i \in \mathbb{Z}_{n}$ by

$$
L_{i}^{\alpha, \beta}(x):=\frac{\pi(x)}{\left(x-x_{i}^{\alpha, \beta}\right) \pi^{\prime}\left(x_{i}^{\alpha, \beta}\right)}, \quad x \in I .
$$

Let $P_{n}$ be the set of all polynomials of degree not more than $n$; clearly,

$$
P_{n}=\operatorname{span}\left\{L_{i}^{\alpha, \beta}: i \in \mathbb{Z}_{n}\right\}
$$

We use the notion $C(I)$ to denote the set of all continuous functions on $I$, equipped with the norm

$$
\|v\|_{\infty}:=\max _{x \in I}|v(x)| .
$$

For $s \in I$, we define a linear functional $\delta_{s}$ on $C(I)$ such that, for any $v \in C(I)$,

$$
\left\langle\delta_{s}, v\right\rangle:=v(s)
$$

The collocation method for solving (3) is to seek a vector $\mathbf{u}:=$ $\left[a_{i}: i \in \mathbb{Z}_{n}\right]^{T}$ such that

$$
u_{n}(x):=\sum_{i \in \mathbb{Z}_{n}} a_{i} L_{i}^{\alpha, \beta}(x), \quad x \in I
$$

satisfies

$$
\left\langle\delta_{x_{j}^{\alpha, \beta}},(\mathscr{I}+\mathscr{K}) u_{n}\right\rangle=\left\langle\delta_{x_{j}^{\alpha, \beta}}, f\right\rangle, \quad j \in \mathbb{Z}_{n} .
$$

The above equation can be rewritten as

$$
a_{i}+\sum_{j \in \mathbb{Z}_{n}} a_{j} \int_{-1}^{x_{i}^{\alpha, \beta}} k\left(x_{i}^{\alpha, \beta}, t\right) L_{j}^{\alpha, \beta}(t) d t=f\left(x_{i}^{\alpha, \beta}\right), \quad i \in \mathbb{Z}_{n} .
$$

For $v \in C(I)$, we define the interpolating operator $\mathscr{L}_{n}^{\alpha, \beta}$ : $C(I) \rightarrow P_{n}$ by

$$
\left(\mathscr{L}_{n}^{\alpha, \beta} v\right)\left(x_{i}^{\alpha, \beta}\right)=v\left(x_{i}^{\alpha, \beta}\right), \quad i \in \mathbb{Z}_{n} .
$$

It it well known that $\mathscr{L}_{n}^{\alpha, \beta} v$ is written as the form

$$
\left(\mathscr{L}_{n}^{\alpha, \beta} v\right)(x)=\sum_{i \in \mathbb{Z}_{n}} v\left(x_{i}\right) L_{i}^{\alpha, \beta}(x), \quad x \in I .
$$

Using these notations we can reformulate (12) into an operator form

$$
\left(\mathscr{I}+\mathscr{L}_{n}^{\alpha, \beta} \mathscr{K}\right) u_{n}=\mathscr{L}_{n}^{\alpha, \beta} f
$$

The difficulty in solving the linear system (12) is to compute the integral term in (12), accurately. In this paper, we adopt the numerical integration rule proposed in [11] to overcome this difficulty. For this purpose, we introduce a simple linear transformation

$$
t=g(x, \tau):=\frac{x+1}{2} \tau+\frac{x-1}{2},
$$

which transfers the integral operator $\mathscr{K}$ into the following form:

$$
(\mathscr{K} v)(x)=\frac{x+1}{2} \int_{I} k(x, g(x, \tau)) v(g(x, \tau)) d \tau .
$$

Then, by using $N+1$-point Legendre-Gauss quadrature formula relative to the Legendre weight $w_{i}, i \in \mathbb{Z}_{N}$, we can obtain the discrete integral operator $\mathscr{K}_{N}$ as follows:

$$
\left(\mathscr{K}_{N} v\right)(x):=\frac{x+1}{2} \sum_{i \in \mathbb{Z}_{N}} w_{i} k\left(x, g\left(x, x_{i}^{0,0}\right)\right) v\left(g\left(x, x_{i}^{0,0}\right)\right) .
$$

Thus, using those notations, a fully discrete spectral Jacobi-collocation method for solving (3) is to seek a vector $\widetilde{\mathbf{u}}:=\left[\widetilde{a}_{i}: i \in \mathbb{Z}_{n}\right]^{T}$ such that

$$
\tilde{u}_{n}(x):=\sum_{i \in \mathbb{Z}_{n}} \tilde{a}_{i} L_{i}^{\alpha, \beta}(x), \quad x \in I,
$$


satisfying

$$
\left(\mathscr{I}+\mathscr{L}_{n}^{\alpha, \beta} \mathscr{K}_{N}\right) \widetilde{u}_{n}=\mathscr{L}_{n}^{\alpha, \beta} f
$$

It is easy to show that the operator equation (20) has the following form:

$$
\begin{aligned}
\tilde{a}_{i} & +\frac{x_{i}^{\alpha, \beta}+1}{2} \\
& \times \sum_{j \in \mathbb{Z}_{n}} \tilde{a}_{j} \sum_{l \in \mathbb{Z}_{N}} w_{l} k\left(x_{i}^{\alpha, \beta}, g\left(x_{i}^{\alpha, \beta}, x_{l}^{0,0}\right)\right) L_{j}^{\alpha, \beta}\left(g\left(x_{i}^{\alpha, \beta}, x_{l}^{0,0}\right)\right) \\
= & f\left(x_{i}^{\alpha, \beta}\right), \quad i \in \mathbb{Z}_{n} .
\end{aligned}
$$

In [11], for the case $N=n$, based on the Gronwall' inequality, Tang et al. analyze the convergence of a spectral Jacobi-collocation method for solving (3) in both $C(I)$ and weighted $L_{w^{0,0}}^{2}(I)$ spaces. However, the stability analysis of the spectral method is not given. Moreover, we observe that the convergence order of the approximate solution in the space $L_{w^{0,0}}^{2}(I)$ is not optimal. Hence, the purpose of this paper is to illustrate that for sufficiently large $n$ and $N$, the operator $\mathscr{I}+\mathscr{L}_{n}^{\alpha, \beta} \mathscr{K}_{N}: P_{n} \rightarrow P_{n}$ has a uniformly bounded inversion in both $C(I)$ and $L_{w^{\alpha, \beta}}^{2}(I)$ spaces, respectively. Moreover, we also show that the approximate solution $\widetilde{u}_{n}$ attains at the most possible convergent order.

\section{Some Preliminaries and Useful Results}

In this section, we will introduce some technical results, which contribute to analyze the stability and convergence on the spectral Jacobi-collocation method for solving (3). To this end, for $i \in \mathbb{N}_{0}$, we use the notation $\mathscr{D}_{x}^{i}$ to denote the $i$ th differential operator on the variable $x$. For $r \in \mathbb{N}$, we introduce the nonuniformly weighted Sobolev space $H_{w^{\alpha, \beta}}^{r}(I)$ by

$$
H_{w^{\alpha, \beta}}^{r}(I):=\left\{v: \mathscr{D}_{x}^{i} v \in L_{w^{\alpha+i, \beta+i}}^{2}(I), i \in \mathbb{Z}_{r}\right\} .
$$

It follows from [19] that there exists a positive constant $\gamma_{1}$ independent of $n$ such that, for $v \in H_{w^{\alpha, \beta}}^{r}(I)$ and $i \in \mathbb{Z}_{r}$,

$$
\left\|\mathscr{D}_{x}^{i}\left(v-\mathscr{L}_{n}^{\alpha, \beta} v\right)\right\|_{w^{\alpha+i, \beta+i}} \leq \gamma_{1}\left\|\mathscr{D}_{x}^{r} v\right\|_{w^{\alpha+r, \beta+r}} n^{i-r},
$$

which implies that

$$
\left\|\mathscr{D}_{x}^{i}\left(\mathscr{L}_{n}^{\alpha, \beta} v\right)\right\|_{w^{\alpha+i, \beta+i}} \leq \gamma_{1}\left\|\mathscr{D}_{x}^{r} v\right\|_{w^{\alpha+r, \beta+r}}+\left\|\mathscr{D}_{x}^{i} v\right\|_{w^{\alpha+i, \beta+i}} .
$$

Moreover, we have the following.

Lemma 1. Suppose that $-1<\alpha_{1}, \beta_{1}<1$. If the parameters $\alpha, \beta$ satisfy the next conditions:

$$
-\frac{1}{2}+\alpha_{1}<\alpha<\frac{3}{2}+\alpha_{1}, \quad-\frac{1}{2}+\beta_{1}<\beta<\frac{3}{2}+\beta_{1},
$$

then there exists a positive constant $\gamma_{2}$ independent of $n$ such that, for $v \in H_{w^{\alpha, \beta}}^{r}(I) \cap C(I)$,

$$
\left\|v-\mathscr{L}_{n}^{\alpha_{1}, \beta_{1}} v\right\|_{w^{\alpha, \beta}} \leq \gamma_{2}\left\|\mathscr{D}_{x}^{r} v\right\|_{w^{\alpha+r, \beta+r}} n^{-r}
$$

Proof. This is a consequence of Theorem 3.4 and (3.13)-(3.14) in [20].

For $r \in \mathbb{N}, i \in \mathbb{Z}_{r}$, the binomial coefficients are given by

$$
\mathrm{C}_{r}^{i}:=r(r-1) \cdots(r-i+1)
$$

We use the notation $C^{r}(I)$ to denote the set of all functions whose $r$ th derivative is continuous on $I$, endowed with the usual norm

$$
\|v\|_{r}:=\sum_{i \in \mathbb{Z}_{r}}\left\|\mathscr{D}_{x}^{i} v\right\|_{\infty} \cdot
$$

For $r_{1}, r_{2} \in \mathbb{N}_{0}$, the notation $C^{r_{1}, r_{2}}\left(I^{2}\right)$ is used to denote the set of all functions such that, for $v \in C^{r_{1}, r_{2}}\left(I^{2}\right), \mathscr{D}_{x}^{r_{1}} \mathscr{D}_{y}^{r_{2}} v$ is continuous on $I^{2}$. Let

$$
\left\|\mathscr{D}_{x}^{r_{1}} \mathscr{D}_{y}^{r_{2}} v\right\|_{\infty}:=\max _{(x, y) \in I^{2}}\left|\mathscr{D}_{x}^{r_{1}} \mathscr{D}_{y}^{r_{2}} v(x, y)\right| .
$$

Next we consider the difference between $\mathscr{K} v$ and $\mathscr{K}_{N} v$.

Lemma 2. Assume that the kernel function $k \in C^{0, m}\left(I^{2}\right)$ for $m \in \mathbb{N}$. If two parameters $\alpha$ and $\beta$ satisfy the conditons

$$
-\frac{1}{2}<\alpha, \beta<\frac{3}{2}, \quad \alpha+\beta \leq 1,
$$

then there exists a positive constant $\gamma_{3}$ independent of $N$ such that when $v \in C^{m}(I)$,

$$
\left\|\mathscr{K} v-\mathscr{K}_{N} v\right\|_{\infty} \leq \gamma_{3}\left(\sum_{i \in \mathbb{Z}_{m}}\left\|\mathscr{D}_{x}^{i} v\right\|_{w^{\alpha+i, \beta+i}}\right) N^{-m} .
$$

Proof. First of all, by setting

$$
\begin{aligned}
b(x, \tau) & :=k(x, g(x, \tau)) v(g(x, \tau)) \\
\left(\mathscr{L}_{N}^{0,0} b\right)(x, \tau) & :=\sum_{i \in \mathbb{Z}_{N}} b\left(x, x_{i}^{0,0}\right) L_{i}^{0,0}(\tau), \quad x, \tau \in I,
\end{aligned}
$$

the integral operator $\mathscr{K}_{N}$ is written as

$$
\left(\mathscr{K}_{N} v\right)(x)=\frac{x+1}{2} \int_{I}\left(\mathscr{L}_{N}^{0,0} b\right)(x, \tau) d \tau, \quad x \in I .
$$

In addition, using the hypothesis that $k \in C^{0, m}\left(I^{2}\right)$ and $v \in$ $C^{m}(I)$ implies that $b \in C^{0, m}\left(I^{2}\right)$. Thus, we write the difference between $\mathscr{K} v$ and $\mathscr{K}_{N} v$ as follows:

$$
\begin{aligned}
& (\mathscr{K} v)(x)-\left(\mathscr{K}_{N} v\right)(x) \\
& =\frac{x+1}{2} \int_{I}\left(w^{-\alpha / 2,-\beta / 2}(\tau)\right) \\
& \quad \times\left(w^{\alpha / 2, \beta / 2}(\tau)\left(b(x, \tau)-\left(\mathscr{L}_{N}^{0,0} b\right)(x, \tau)\right)\right) d \tau .
\end{aligned}
$$

Employing Cauchy-Schwartz inequality to the right hand side of the above equation and then using the result (26) with 
$\alpha_{1}:=0, \beta_{1}:=0, n:=N$ and $r:=m$ produce that there exists a positive constant $\xi$ independent of $N$,

$$
\left|(\mathscr{K} v)(x)-\left(\mathscr{K}_{N} v\right)(x)\right|^{2} \leq \xi G(x)\|1\|_{w^{-\alpha,-\beta}} N^{-2 m},
$$

where $G(x)$ is given by

$$
\begin{array}{r}
G(x):=\left(\frac{1+x}{2}\right)^{2} \int_{I} w^{\alpha+m, \beta+m}(\tau)\left(\left(\mathscr{D}_{\tau}^{m} b\right)(x, \tau)\right)^{2} d \tau, \\
x \in I .
\end{array}
$$

It remains to estimate $G(x)$. A direct computation leads to

$$
\begin{aligned}
& G(x) \\
& =\left(\frac{1+x}{2}\right)^{2 m+2} \\
& \quad \times \int_{I} w^{\alpha+m, \beta+m}(\tau) \\
& \quad \times\left(\sum_{i \in \mathbb{Z}_{m}} \mathrm{C}_{m}^{i}\left(\mathscr{D}_{g}^{i} v\right)(g(x, \tau))\left(\mathscr{D}_{g}^{m-i} k\right)(x, g(x, \tau))\right)^{2} d \tau .
\end{aligned}
$$

Making use of a linear transform $t:=g(x, \tau)$ to the right hand side of the above equation produces

$$
\begin{aligned}
& G(x) \\
& =\left(\frac{1+x}{2}\right)^{1-\alpha-\beta} \\
& \quad \times \int_{-1}^{x}(1+t)^{\alpha+m}(x-t)^{\beta+m} \\
& \quad \times\left(\sum_{i \in \mathbb{Z}_{m}} \mathrm{C}_{m}^{i}\left(\mathscr{D}_{t}^{i} v\right)(t)\left(\mathscr{D}_{t}^{m-i} k\right)(x, t)\right)^{2} d t .
\end{aligned}
$$

Using the discrete Cauchy Schwartz inequality into the right hand side of the above equation obtains

$$
\begin{aligned}
G(x) \leq \max _{i \in \mathbb{Z}_{m}}\left\|\mathscr{D}_{t}^{i} k\right\|_{\infty}^{2}\left(\sum_{i \in \mathbb{Z}_{m}}\left(C_{n}^{i}\right)^{2}\right)\left(\frac{1+x}{2}\right)^{1-\alpha-\beta} \\
\quad \times\left(\sum_{i \in \mathbb{Z}_{m}} \int_{-1}^{x}(1+t)^{\alpha+m}(x-t)^{\beta+m}\left(\mathscr{D}_{t}^{i} v\right)^{2}(t) d t\right),
\end{aligned}
$$

where combining the fact that $\alpha+\beta \leq 1$ leads to

$$
G(x) \leq \max _{i \in \mathbb{Z}_{m}}\left\|\mathscr{D}_{t}^{i} k\right\|_{\infty}^{2}\left(\sum_{i \in \mathbb{Z}_{m}}\left(C_{n}^{i}\right)^{2}\right)\left(\sum_{i \in \mathbb{Z}_{m}}\left\|\mathscr{D}_{t}^{i} v\right\|_{w^{\alpha+i, \beta+i}}^{2}\right) .
$$

Substituting the above estimate on $G(x)$ into the right hand side of (35) yields the desired conclusion (31) with $\gamma_{3}$ being given by

$$
\gamma_{3}:=\max _{i \in \mathbb{Z}_{m}}\left\|\mathscr{D}_{t}^{i} k\right\|_{\infty}\left(\xi\|1\|_{w^{-\alpha,-\beta}} \sum_{i \in \mathbb{Z}_{m}}\left(C_{m}^{i}\right)^{2}\right)^{1 / 2} .
$$

Using Lemma 2, we can obtain the following.

Corollary 3. Suppose that the conditions of Lemma 2 hold, then for $v \in P_{n}$, the following two estimates hold:

$$
\begin{gathered}
\left\|\mathscr{K} v-\mathscr{K}_{N} v\right\|_{\infty} \leq 2 \gamma_{3}\|v\|_{w^{\alpha, \beta}}\left(\frac{n}{N}\right)^{m}, \\
\left\|\mathscr{K} v-\mathscr{K}_{N} v\right\|_{\infty} \leq 2 \gamma_{3}\|1\|_{w^{\alpha, \beta}}\|v\|_{\infty}\left(\frac{n}{N}\right)^{m} .
\end{gathered}
$$

Proof. We observe that if (42) holds, then by using the fact

$$
\|v\|_{w^{\alpha, \beta}} \leq\|1\|_{w^{\alpha, \beta}}\|v\|_{\infty}, \quad v \in P_{n}
$$

we can easily obtain the result (43). Thus, we only require to estimate (42). In fact, by using the inverse inequality relative to two norms weighted with different Jacobi weight functions in Theorem 3.31 in [19], there exists a positive constant $\xi$ independent of $n$ such that, for $v \in P_{n}$ and $i \in \mathbb{Z}_{m}$,

$$
\left\|\mathscr{D}_{t}^{i} v\right\|_{w^{\alpha+i, \beta+i}} \leq \xi n\left\|\mathscr{D}_{t}^{i-1} v\right\|_{w^{\alpha+i-1, \beta+i-1}} \text {. }
$$

By the above inequality, we can obtain that

$$
\sum_{i \in \mathbb{Z}_{m}}\left\|\mathscr{D}_{t}^{i} v\right\|_{w^{\alpha+i, \beta+i}} \leq \sum_{i \in \mathbb{Z}_{m}}(\xi n)^{i}\|v\|_{w^{\alpha, \beta}}
$$

where combining (31) yields the desired conclusion (42).

\section{The Stability and Convergence Analysis under the $L^{\infty}$ Norm}

In this section, we will establish that, for sufficiently large $n$ and $N$, the operator $\mathscr{I}+\mathscr{L}_{n}^{\alpha, \beta} \mathscr{K}_{N}: P_{n} \rightarrow P_{n}$ has a uniformly bounded inversion in the space $C(I)$ and then show that the approximate solution $\widetilde{u}_{n}$ arrives at the most possible convergent order under the $L^{\infty}$ norm. To this end, we first give some notations. For $r \in \mathbb{N}_{0}$ and $\nu \in(0,1]$, the notation $H^{r, \nu}(I)$ is used to denote the space of functions whose $r$ th derivative is Hölder continuous on $I$ with exponent $\nu$. The norm of the space is defined by

$$
\|v\|_{r, v}:=\|v\|_{r}+\sup _{x, y \in I, x \neq y} \frac{\left|\left(\mathscr{D}_{x}^{r} v\right)(x)-\left(\mathscr{D}_{y}^{r} v\right)(y)\right|}{|x-y|^{v}} .
$$

Lemma 4. Suppose that the kernel function $k \in C^{1,0}\left(I^{2}\right)$; then the operator $\mathscr{K}$ is a bounded linear operator from $C(I)$ to $H^{0,1}(I)$; that is, for $v \in C(I)$,

$$
\|\mathscr{K} v\|_{0,1} \leq\left(4\|k\|_{\infty}+2\left\|\mathscr{D}_{x}^{1} k\right\|_{\infty}\right)\|v\|_{\infty} .
$$


Moreover, for $-1<\alpha, \beta<1$, the operator $\mathscr{K}: L_{w^{\alpha, \beta}}^{2}(I) \rightarrow$ $C(I)$ is also a linear bounded operator; that is, for $v \in L_{w^{\alpha, \beta}}^{2}(I)$,

$$
\|\mathscr{K} v\|_{\infty} \leq\|k\|_{\infty}\|1\|_{w^{-\alpha,-\beta}}^{1 / 2}\|v\|_{w^{\alpha, \beta}}
$$

Proof. It is easily proved that the operator $\mathscr{K}$ is a linear operator from the space $C(I)$ to the space $H^{0,1}(I)$ or from $L_{w^{\alpha, \beta}}^{2}(I)$ to $C(I)$.

Next we illustrate that (48) holds. By the definition of the norm,

$$
\|\mathscr{K} v\|_{\infty} \leq\|v\|_{\infty} \max _{x \in I} \int_{-1}^{x}|k(x, t)| d t
$$

which implies that

$$
\|\mathscr{K} v\|_{\infty} \leq 2\|k\|_{\infty}\|v\|_{\infty} .
$$

On the other hand, for all $x_{1}, x_{2} \in I$, by introducing

$$
\begin{gathered}
I_{1}:=\int_{-1}^{x_{1}}\left(k\left(x_{1}, t\right)-k\left(x_{2}, t\right)\right) v(t) d t \\
I_{2}:=\int_{x_{2}}^{x_{1}} k\left(x_{2}, t\right) v(t) d t
\end{gathered}
$$

we can obtain that

$$
(\mathscr{K} v)\left(x_{1}\right)-(\mathscr{K} v)\left(x_{2}\right)=I_{1}+I_{2},
$$

where using the triangle inequality yields that

$$
\left|(\mathscr{K} v)\left(x_{1}\right)-(\mathscr{K} v)\left(x_{2}\right)\right| \leq\left|I_{1}\right|+\left|I_{2}\right| .
$$

The left thing is to give an estimation of $I_{1}$ and $I_{2}$. First, employing Lagrange midvalue differential theorem to $I_{1}$ yields that

$$
\left|I_{1}\right| \leq 2\left\|\mathscr{D}_{x}^{1} k\right\|_{\infty}\|v\|_{\infty}\left|x_{1}-x_{2}\right|
$$

A direct estimation for $I_{2}$ produces that

$$
\left|I_{2}\right| \leq 2\|k\|_{\infty}\|v\|_{\infty}\left|x_{1}-x_{2}\right| .
$$

Thus, substituting the estimates (55)-(56) into the right hand side of (54) leads that

$$
\begin{aligned}
& \left|(\mathscr{K} v)\left(x_{1}\right)-(\mathscr{K} v)\left(x_{2}\right)\right| \\
& \quad \leq 2\left(\|k\|_{\infty}+\left\|\mathscr{D}_{x}^{1} k\right\|_{\infty}\right)\|v\|_{\infty}\left|x_{1}-x_{2}\right|,
\end{aligned}
$$

where (51) yields the desired conclusion (48).

In the following we show that the result (49) holds. Noticing,

$$
\begin{aligned}
& \|\mathscr{K} v\|_{\infty} \\
& =\max _{x \in I}\left|\int_{-1}^{x} k(x, t) v(t) d t\right| \\
& =\max _{x \in I}\left|\int_{-1}^{x}\left(w^{-\alpha / 2,-\beta / 2}(t) k(x, t)\right)\left(w^{\alpha / 2, \beta / 2}(t) v(t)\right) d t\right| .
\end{aligned}
$$

Using Cauchy-Schwartz inequality to the right hand side of the equation above yields

$$
\|\mathscr{K} v\|_{\infty}^{2} \leq \max _{x \in I} \int_{-1}^{x}\left(w^{-\alpha,-\beta}(t) k^{2}(x, t)\right)\|v\|_{w^{\alpha, \beta}}^{2},
$$

which implies that

$$
\|\mathscr{K} v\|_{\infty}^{2} \leq\|k\|_{\infty}^{2}\|1\|_{w^{-\alpha,-\beta}}\|v\|_{w^{\alpha, \beta}}^{2}
$$

This complete the proof of (49).

The next result concerns on the bound of the norm $\left\|\mathscr{K} v-\mathscr{L}_{n}^{\alpha, \beta} \mathscr{K} v\right\|_{\infty}$ for $v \in C(I)$. For this purpose, we introduce the result on the Lebesgue constant corresponding to the Lagrange interpolation polynomials associated with the zeros of the Jacobi polynomials, which comes from Lemma 3.4 in [5]:

$$
\begin{aligned}
\left\|\mathscr{L}_{n}^{\alpha, \beta}\right\|_{\infty} & =\max _{\|v\|_{\infty}=1}\left\|\mathscr{L}_{n}^{\alpha, \beta} v\right\|_{\infty} \\
& = \begin{cases}\mathcal{O}(\log n), & -1<\alpha, \beta \leq-\frac{1}{2}, \\
\mathcal{O}\left(n^{(1 / 2)+\max \{\alpha, \beta\}}\right), & \text { otherwise. }\end{cases}
\end{aligned}
$$

Further, we also require to make use of another result of Ragozin, coming from [21, 22], which states that, for any $v \in H^{r, v}(I)$, there exist a polynomial $q \in P_{n}$ and a positive constant $\varsigma_{1}$ such that

$$
\|v-q\|_{\infty} \leq \varsigma_{1} n^{-r-v}\|v\|_{r, v}
$$

A combination of (61) and (62) leads to that there exists a positive constant $\varsigma_{2}$ such that

$$
\begin{aligned}
& \left\|v-\mathscr{L}_{n}^{\alpha, \beta} v\right\|_{\infty} \\
& \leq \varsigma_{2}\|v\|_{r, v} \begin{cases}n^{-r-v} \log n, & -1<\alpha, \beta \leq-\frac{1}{2}, \\
n^{(1 / 2)+\max \{\alpha, \beta\}-r-v}, & \text { otherwise. }\end{cases}
\end{aligned}
$$

Lemma 5. Suppose that the kernel function $k \in C^{1,0}\left(I^{2}\right)$. Then there exists a positive constant $\varsigma_{3}$ independent of $n$ such that when $v \in C(I)$,

$$
\begin{aligned}
& \left\|\mathscr{K} v-\mathscr{L}_{n}^{\alpha, \beta} \mathscr{K} v\right\|_{\infty} \\
& \quad \leq \varsigma_{3}\|v\|_{\infty} \begin{cases}n^{-1} \log n, & -1<\alpha, \beta \leq-\frac{1}{2}, \\
n^{-(1 / 2)+\max \{\alpha, \beta\}}, & \text { otherwise. }\end{cases}
\end{aligned}
$$

Proof. It follows from Lemma 4 that $\mathscr{K} v \in H^{0,1}(I)$ for $v \in$ $C(I)$, where combining (63) obtains that there exists a positive constant $\xi$ independent of $n$ :

$$
\begin{aligned}
& \left\|\mathscr{K} v-\mathscr{L}_{n}^{\alpha, \beta} \mathscr{K} v\right\|_{\infty} \\
& \quad \leq \xi\|\mathscr{K} v\|_{0,1} \begin{cases}n^{-1} \log n, & -1<\alpha, \beta \leq-\frac{1}{2}, \\
n^{-(1 / 2)+\max \{\alpha, \beta\}}, & \text { otherwise. }\end{cases}
\end{aligned}
$$


Substituting the estimate (48) into the right hand side of the above equation yields the desired conclusion with $\varsigma_{3}$ being given by

$$
\varsigma_{3}:=\xi\left(4\|k\|_{\infty}+2\left\|\mathscr{D}_{x}^{1} k\right\|_{\infty}\right)
$$

We use the notation $[x]$ to denote the largest integer not more than $x$. Moreover, by Theorem 3.10 in [23], if the kernel function $k$ is a smooth function, the operator $\mathscr{I}+\mathscr{K}: C(I) \rightarrow$ $C(I)$ has a bounded inversion; that is, for any $v \in P_{n}$, there exists a positive constant $\rho$ such that

$$
\|(\mathscr{I}+\mathscr{K}) v\|_{\infty} \geq \rho\|v\|_{\infty} .
$$

Theorem 6. Suppose that $k \in C^{1, m}\left(I^{2}\right),-1 / 2<\alpha, \beta<1 / 2$. If we choose $N$ as follows:

$$
N \geq N_{\min }:=\left[n^{1+(1 / 2 m)+(\min \{\alpha, \beta\} / m)} \log ^{1 / m} n\right]+1,
$$

then there exists a positive integer $n_{0}$ such that when $n \geq n_{0}$ and for $v \in P_{n}$,

$$
\left\|\left(\mathscr{I}+\mathscr{L}_{n}^{\alpha, \beta} \mathscr{K}_{N}\right) v\right\|_{\infty} \geq \frac{\rho}{2}\|v\|_{\infty},
$$

where $\rho$ appears in (67).

Proof. It follows from the hypothesis that $-1 / 2<\alpha, \beta<1 / 2$ that $n^{-(1 / 2)+\max \{\alpha, \beta\}}$ tends to zero as $n$ tends to $\infty$. Hence, using (64) there exists a positive integer $n_{1}$ such that $n \geq n_{1}$,

$$
\left\|\mathscr{K} v-\mathscr{L}_{n}^{\alpha, \beta} v\right\|_{\infty} \leq \frac{\rho}{4}\|v\|_{\infty} .
$$

On the other hand, using (61) with the hypothesis that $-1 / 2<\alpha, \beta<1 / 2$ yields that there exists a positive constant $\xi_{1}$ such that, for $v \in P_{n}$,

$$
\left\|\mathscr{L}_{n}^{\alpha, \beta} \mathscr{K} v-\mathscr{L}_{n}^{\alpha, \beta} \mathscr{K}_{N} v\right\|_{\infty} \leq \xi_{1}\left\|\mathscr{K} v-\mathscr{K}_{N} v\right\|_{\infty} n^{(1 / 2)+\max \{\alpha, \beta\}},
$$

where combining (43) and (68) produces that there exists a positive constant $\xi_{2}$,

$$
\left\|\mathscr{L}_{n}^{\alpha, \beta} \mathscr{K} v-\mathscr{L}_{n}^{\alpha, \beta} \mathscr{K}_{N} v\right\|_{\infty} \leq \xi_{2}\|v\|_{\infty} \log ^{-1} n .
$$

Similarly as before, by the fact that $\log ^{-1} n$ tends to 0 as $n$ tends to $\infty$, there exists a positive integer $n_{2}$ such that, for $n \geq n_{2}$,

$$
\left\|\mathscr{L}_{n}^{\alpha, \beta} \mathscr{K} v-\mathscr{L}_{n}^{\alpha, \beta} \mathscr{K}_{N} v\right\|_{\infty} \leq \frac{\rho}{4}\|v\|_{\infty} .
$$

Hence, when $n \geq n_{0}:=\max \left\{n_{1}, n_{2}\right\}$, combining these three estimates (67), (70), and (73) yields that

$$
\begin{aligned}
\|(\mathscr{I} & \left.+\mathscr{L}_{n}^{\alpha, \beta} \mathscr{K}_{N}\right) v \|_{\infty} \\
\geq & \|v+\mathscr{K} v\|_{\infty}-\left\|\mathscr{K} v-\mathscr{L}_{n}^{\alpha, \beta} \mathscr{K} v\right\|_{\infty} \\
& -\left\|\mathscr{L}_{n}^{\alpha, \beta} \mathscr{K} v-\mathscr{L}_{n}^{\alpha, \beta} \mathscr{K}_{N} v\right\|_{\infty} \\
\geq & \frac{\rho}{2}\|v\|_{\infty},
\end{aligned}
$$

proving the desired conclusion (69).
Theorem 6 ensures that, for sufficient large $n$, the operator equation (20) has a unique solution $\widetilde{u}_{n}$. The next result considers the convergent order of the approximate solution $\tilde{u}_{n}$ in $L^{\infty}$ norm.

Theorem 7. Suppose that the kernel function $k \in C^{m, m}\left(I^{2}\right)$, $f \in C^{m}(I)$, and $-1 / 2<\alpha, \beta<1 / 2$. If we choose $N$ as in (68), then there exist a positive constant $\eta$ and a positive integer $n_{0}$ such that, for $n \geq n_{0}$,

$$
\left\|u-\widetilde{u}_{n}\right\|_{\infty} \leq \eta\|u\|_{m} n^{(1 / 2)+\max \{\alpha, \beta\}-m} .
$$

Proof. We first notice that it follows from the hypothesis that $k \in C^{m, m}\left(I^{2}\right)$ and $f \in C^{m}(I)$ that (3) has a unique solution $u \in C^{m}(I)$, which implies that $u \in H^{m-1,1}(I)$. By using the triangle inequality,

$$
\left\|u-\widetilde{u}_{n}\right\|_{\infty} \leq\left\|u-\mathscr{L}_{n}^{\alpha, \beta} u\right\|_{\infty}+\left\|\mathscr{L}_{n}^{\alpha, \beta} u-\widetilde{u}_{n}\right\|_{\infty} .
$$

Upon the estimation (63) with $v:=u$, we only require to estimate the second term in the right hand side of the above equation. In fact, employing $\mathscr{L}_{n}^{\alpha, \beta}$ to both sides of (3) yields that

$$
\mathscr{L}_{n}^{\alpha, \beta} u+\mathscr{L}_{n}^{\alpha, \beta} \mathscr{K} u=\mathscr{L}_{n}^{\alpha, \beta} f .
$$

A direct computation of the above equation and (20) confirms that

$$
\begin{aligned}
& \left(\mathscr{I}+\mathscr{L}_{n}^{\alpha, \beta} \mathscr{K}_{N}\right)\left(\tilde{u}_{n}-\mathscr{L}_{n}^{\alpha, \beta} u\right) \\
& =\mathscr{L}_{n}^{\alpha, \beta} \mathscr{K} u-\mathscr{L}_{n}^{\alpha, \beta} \mathscr{K}_{N} \mathscr{L}_{n}^{\alpha, \beta} u .
\end{aligned}
$$

By Theorem 6, there exists a positive integer $n_{0}$ such that $n \geq n_{0}$,

$$
\left\|\widetilde{u}_{n}-\mathscr{L}_{n}^{\alpha, \beta} u\right\|_{\infty} \leq \frac{2}{\rho}\left\|\mathscr{L}_{n}^{\alpha, \beta} \mathscr{K} u-\mathscr{L}_{n}^{\alpha, \beta} \mathscr{K}_{N} \mathscr{L}_{n}^{\alpha, \beta} u\right\|_{\infty},
$$

where combining (61) leads that there exists a positive constant $\xi_{1}$ such that

$$
\left\|\tilde{u}_{n}-\mathscr{L}_{n}^{\alpha, \beta} u\right\|_{\infty} \leq \xi_{1}\left\|\mathscr{K} u-\mathscr{K}_{N} \mathscr{L}_{n}^{\alpha, \beta} u\right\|_{\infty} n^{(1 / 2)+\max \{\alpha, \beta\}} .
$$

To obtain the estimation of the right hand side of equation (80), we let

$$
\begin{gathered}
I_{1}:=\left\|\mathscr{K} u-\mathscr{K} \mathscr{L}_{n}^{\alpha, \beta} u\right\|_{\infty}, \\
I_{2}:=\left\|\mathscr{K} \mathscr{L}_{n}^{\alpha, \beta} u-\mathscr{K}_{N} \mathscr{L}_{n}^{\alpha, \beta} u\right\|_{\infty} .
\end{gathered}
$$

Clearly,

$$
\left\|\mathscr{K} u-\mathscr{K}_{N} \mathscr{L}_{n}^{\alpha, \beta} u\right\|_{\infty} \leq I_{1}+I_{2} .
$$

It remains to estimate $I_{1}$ and $I_{2}$, respectively. First, using the hypothesis that $-1 / 2<\alpha, \beta<1 / 2$ and the result (49) with $v:=u-\mathscr{L}_{n}^{\alpha, \beta} u$ produces that there exists a positive constant $\xi_{2}$ independent of $n$ such that

$$
I_{1} \leq \xi_{2}\left\|u-\mathscr{L}_{n}^{\alpha, \beta} u\right\|_{w^{\alpha, \beta}},
$$


where combining the result (23) with $i:=0, r:=m, v:=u$ yields that there exists a positive constant $\xi_{3}$ independent of $n$ such that

$$
I_{1} \leq \xi_{3}\left\|\mathscr{D}_{x}^{m} u\right\|_{w^{\alpha+m, \beta+m}} n^{-m}
$$

Hence, a combination of (84) and the following inequality

$$
\left\|\mathscr{D}_{x}^{l} u\right\|_{w^{\alpha+l, \beta+l}} \leq\|1\|_{w^{\alpha, \beta}}\left\|\mathscr{D}_{x}^{l} u\right\|_{\infty}, \quad l \in \mathbb{N},
$$

produces that there exists a positive constant $\xi_{4}$ such that

$$
I_{1} \leq \xi_{4}\|u\|_{m} n^{-m}
$$

On the other hand, using the results (24) and (31) leads that there exists a positive constant $\xi_{5}$ such that

$$
I_{2} \leq \xi_{5}\left(\sum_{i \in \mathbb{Z}_{m}}\left\|\mathscr{D}_{x}^{i} u\right\|_{w^{\alpha+i, \beta+i}}\right) N^{-m},
$$

where combining (68) and (85) yields that there exists a positive constant $\xi_{6}$,

$$
I_{2} \leq \xi_{6}\|u\|_{m} n^{-m-(1 / 2)-\max \{\alpha, \beta\}} \log ^{-1} n .
$$

A combination of the above estimation and (80), (82), and (86) yields the desired result.

Theorem 7 illustrates that the approximate solution obtained by the proposed method arrives at the most possible convergent order.

\section{The Stability and Convergence Analysis under the $L_{w^{\alpha, \beta}}^{2}$ Norm}

As demonstrated in the previous section, in this section we are going to prove that, for sufficiently large $n$ and $N$, the operator $\mathscr{I}+\mathscr{L}_{n}^{\alpha, \beta} \mathscr{K}_{N}: P_{n} \rightarrow P_{n}$ has a uniformly bounded inversion in the $L_{w^{\alpha, \beta}}^{2}(I)$ space and then show that the approximate solution arrives at the optimal convergent order. To this end, we first give a few results.

Lemma 8. Suppose that $-1<\lambda_{1}, \lambda_{2}<1$ and $h \in[0,1]$. If $x, x+h \in I$, then one has that

$$
\int_{x}^{x+h} w^{\lambda_{1}, \lambda_{2}}(t) d t \leq \frac{2 \max \left\{h, h^{1+\min \left\{\lambda_{1}, \lambda_{2}\right\}}\right\}}{1+\min \left\{\lambda_{1}, \lambda_{2}\right\}} .
$$

Proof. We will prove that the result (89) holds in the following four cases: (1) $\lambda_{1} \geq 0$ and $\lambda_{2} \geq 0$; (2) $\lambda_{1} \geq 0$ but $\lambda_{2}<$ 0; (3) $\lambda_{1}<0$ while $\lambda_{2} \geq 0$; (4) $\lambda_{1}<0$ and $\lambda_{2}<0$.

Firstly, we notice that, for $\lambda_{1} \geq 0, \lambda_{2} \geq 0$,

$$
(1-t)^{\lambda_{1}}(1+t)^{\lambda_{2}} \leq 2,
$$

which confirms the desired conclusion.

If the conditions $\lambda_{1}<0$ and $\lambda_{2} \geq 0$ hold, then using

$$
(1+t)^{\lambda_{2}} \leq 2
$$

produces

$$
\int_{x}^{x+h} w^{\lambda_{1}, \lambda_{2}}(t) d t \leq 2 \int_{x}^{x+h}(1-t)^{\lambda_{1}} d t \leq \frac{2 h^{1+\lambda_{1}}}{1+\lambda_{1}}
$$

which ensures the desired conclusion.

In a similar approach as the above case, clearly, the result (89) holds for the case that $\lambda_{1} \geq 0$ while $\lambda_{2}<0$.

At last, when the conditions $\lambda_{1}<0$ and $\lambda_{2}<0$ hold, using the next equation

$$
(1-t)^{\lambda_{1}}(1+t)^{\lambda_{2}}=\frac{(1-t)^{\lambda_{1}}+(1+t)^{\lambda_{2}}}{(1-t)^{-\lambda_{1}}+(1+t)^{-\lambda_{2}}}
$$

can produce

$$
(1-t)^{\lambda_{1}}(1+t)^{\lambda_{2}} \leq(1-t)^{\lambda_{1}}+(1+t)^{\lambda_{2}} .
$$

Thus, again using the same method as before yields the desired result.

Next we ensure that the operator $\mathscr{K}: L_{w^{\alpha, \beta}}^{2}(I) \rightarrow H^{0, \kappa}(I)$ is a bounded linear operator with certain positive constant $\kappa$.

Lemma 9. Suppose that $-1<\alpha, \beta<1, k \in C^{1,0}\left(I^{2}\right)$; then $\mathscr{K}$ is a bounded linear operator from $L_{w^{\alpha, \beta}}^{2}(I)$ into $H^{0, \kappa}(I)$ with $\kappa:=\min \{1 / 2,(1 / 2)+\min \{-\alpha / 2,-\beta / 2\}\} ;$ that is, there exists a positive constant $\zeta_{1}$ such that, for $v \in L_{w^{\alpha, \beta}}^{2}(I)$,

$$
\|\mathscr{K} v\|_{0, \kappa} \leq \zeta_{1}\|v\|_{w^{\alpha, \beta}} .
$$

Proof. By the estimation (49) in Lemma 5, there exists a positive constant $\xi_{1}$ such that, for $v \in L_{w^{\alpha, \beta}}^{2}(I)$,

$$
\|\mathscr{K} v\|_{\infty} \leq \xi_{1}\|v\|_{w^{\alpha, \beta}} .
$$

On the other hand, for $x_{1}, x_{2} \in I$, without loss of generality, we assume that $x_{1} \leq x_{2}$. By introducing

$$
\begin{gathered}
I_{1}:=\int_{-1}^{x_{2}}\left(k\left(x_{1}, t\right)-k\left(x_{2}, t\right)\right) v(t) d t, \\
I_{2}:=\int_{x_{1}}^{x_{2}} k\left(x_{1}, t\right) v(t) d t,
\end{gathered}
$$

we have

$$
(\mathscr{K} v)\left(x_{1}\right)-(\mathscr{K} v)\left(x_{2}\right)=I_{1}+I_{2} .
$$

Hence, it remains to estimate $I_{1}$ and $I_{2}$, respectively. For this purpose, by reformulating $I_{1}$ and $I_{2}$ as follows

$$
\begin{gathered}
I_{1}:=\int_{-1}^{x_{2}}\left(w^{-\alpha / 2,-\beta / 2}(t)\left(k\left(x_{1}, t\right)-k\left(x_{2}, t\right)\right)\right) \\
\quad \times\left(w^{\alpha / 2, \beta / 2}(t) v(t)\right) d t \\
I_{2}:=\int_{x_{1}}^{x_{2}}\left(w^{-\alpha / 2,-\beta / 2}(t) k\left(x_{1}, t\right)\right)\left(w^{\alpha / 2, \beta / 2}(t) v(t)\right) d t
\end{gathered}
$$


and then employing Cauchy-Schwarz inequality to $I_{1}$ and $I_{2}$, respectively, we can obtain that

$$
\begin{gathered}
I_{1}^{2} \leq\left(\int_{-1}^{x_{2}} w^{-\alpha,-\beta}(t)\left(k\left(x_{1}, t\right)-k\left(x_{2}, t\right)\right)^{2} d t\right) \\
\times\left(\int_{-1}^{x_{2}} w^{\alpha, \beta}(t) v^{2}(t) d t\right) \\
I_{2}^{2} \leq\left(\int_{x_{1}}^{x_{2}} w^{-\alpha,-\beta}(t) k^{2}\left(x_{1}, t\right) d t\right)\left(\int_{x_{1}}^{x_{2}} w^{\alpha, \beta}(t) v^{2}(t) d t\right) .
\end{gathered}
$$

Using the hypothesis that $k \in C^{1,0}\left(I^{2}\right)$ and the Lagrange midvalue differential theorem yields that

$$
I_{1}^{2} \leq\left\|\mathscr{D}_{x}^{1} k\right\|_{\infty}^{2}\|1\|_{w^{-\alpha,-\beta}}\|v\|_{w^{\alpha, \beta}}^{2}\left|x_{1}-x_{2}\right|^{2}
$$

A direct estimation for $I_{2}$ produces that

$$
I_{2}^{2} \leq\|k\|_{\infty}^{2}\|v\|_{w^{\alpha, \beta}}^{2} \int_{x_{1}}^{x_{2}} w^{-\alpha,-\beta}(t) d t
$$

If the condition $x_{2}-x_{1}>1$ holds, then we have

$$
I_{2}^{2} \leq\|k\|_{\infty}^{2}\|v\|_{w^{\alpha, \beta}}^{2}\|1\|_{w^{-\alpha,-\beta}}\left|x_{1}-x_{2}\right|^{\kappa},
$$

otherwise, using (102), where combining (89) with $\lambda_{1}:=$ $-\alpha, \lambda_{2}:=-\beta, x:=x_{1}$ and $x+h:=x_{2}$ leads to that there exists a positive constant $\xi_{2}$ such that

$$
I_{2}^{2} \leq \xi_{2}\|v\|_{w^{\alpha, \beta}}^{2} \max \left\{\left|x_{1}-x_{2}\right|,\left|x_{1}-x_{2}\right|^{1+\min \{-\alpha,-\beta\}}\right\} .
$$

A combination of (98)-(104) and the triangle inequality yields that there exists a positive constant $\xi_{3}$ such that

$$
\left|(\mathscr{K} v)\left(x_{1}\right)-(\mathscr{K} v)\left(x_{2}\right)\right| \leq \xi_{3}\|v\|_{w^{\alpha, \beta}}\left|x_{1}-x_{2}\right|^{\kappa}
$$

where (96) draws the desired conclusion.

The next result concerns on difference between $\mathscr{K} v$ and $\mathscr{L}_{n}^{\alpha, \beta} \mathscr{K} v$ for $v \in L_{w^{\alpha, \beta}}^{2}(I)$. For this purpose, we will make use of the next result proposed in [5]. For any $v \in C(I)$, there exists a positive constant $\zeta_{2}$ independent of $n$ :

$$
\left\|\mathscr{L}_{n}^{\alpha, \beta} v\right\|_{w^{\alpha, \beta}} \leq \zeta_{2}\|v\|_{\infty}
$$

A combination of (61) and (106) leads to that there exists a positive constant $\zeta_{3}$ such that, for $v \in H^{r, v}(I)$,

$$
\left\|v-\mathscr{L}_{n}^{\alpha, \beta} v\right\|_{w^{\alpha, \beta}} \leq \zeta_{3}\|v\|_{r, v} n^{-r-v} .
$$

Again using Theorem 3.10 in [23], we know that 0 is the unique eigenvalue of Volterra integral operator $\mathscr{K}$; consequently, the operator $\mathscr{I}+\mathscr{K}: L_{w^{\alpha, \beta}}^{2}(I) \rightarrow L_{w^{\alpha, \beta}}^{2}(I)$ has a bounded inversion; that is, for any $v \in P_{n}$, there exists a positive constant $\varrho$ such that

$$
\|(\mathscr{I}+\mathscr{K}) v\|_{w^{\alpha, \beta}} \geq \varrho\|v\|_{w^{\alpha, \beta}} .
$$

Theorem 10. Suppose that $k \in C^{1, m}\left(I^{2}\right)$ and $-1 / 2<\alpha, \beta<$ $1, \alpha+\beta \leq 1$. If one chooses $N$ as follows:

$$
N \geq N_{\min }:=\left[\mathrm{n} \log ^{1 / \mathrm{m}} \mathrm{n}\right]+1,
$$

then there exists a positive integer $n_{0}$ such that $n \geq n_{0}$ and for $v \in P_{n}$,

$$
\left\|\left(\mathscr{I}+\mathscr{L}_{n}^{\alpha, \beta} \mathscr{K}_{N}\right) v\right\|_{w^{\alpha, \beta}} \geq \frac{\varrho}{2}\|v\|_{w^{\alpha, \beta}}
$$

where $\varrho$ appears in (108).

Proof. This proof is similar to that of Theorem 6. By Lemma 9, for $v \in P_{n}$, we have $\mathscr{K} v \in H^{0, \kappa}(I)$, where combining (95) and (107) obtains that there exists a positive constant $\xi_{1}$,

$$
\left\|\mathscr{K} v-\mathscr{L}_{n}^{\alpha, \beta} \mathscr{K} v\right\|_{w^{\alpha, \beta}} \leq \xi_{1}\|v\|_{w^{\alpha, \beta}} n^{-\kappa} .
$$

Hence, by the fact that $\lim _{n \rightarrow \infty} n^{-\kappa}=0$, there exists a positive integer $n_{1}$ such that, for $n \geq n_{1}$,

$$
\left\|\mathscr{K} v-\mathscr{L}_{n}^{\alpha, \beta} \mathscr{K} v\right\|_{w^{\alpha, \beta}} \leq \frac{\varrho}{4}\|v\|_{w^{\alpha, \beta}} .
$$

On the other hand, using (106) obtains that there exists a positive constant $\xi_{2}$ such that

$$
\left\|\mathscr{L}_{n}^{\alpha, \beta} \mathscr{K} v-\mathscr{L}_{n}^{\alpha, \beta} \mathscr{K}_{N} v\right\|_{w^{\alpha, \beta}} \leq \xi_{2}\left\|\mathscr{K} v-\mathscr{K}_{N} v\right\|_{\infty} .
$$

By the hypothesis that $-1 / 2<\alpha, \beta \leq 1$, and $\alpha+\beta \leq 1$, a combination of (42) and (109) yields that there exists a positive constant $\xi_{3}$ such that

$$
\left\|\mathscr{K} v-\mathscr{K}_{N} v\right\|_{\infty} \leq \xi_{3}\|v\|_{w^{\alpha, \beta}} \log ^{-1} n .
$$

Substituting the above estimation into the right hand side of (113) produces that

$$
\left\|\mathscr{L}_{n}^{\alpha, \beta} \mathscr{K} v-\mathscr{L}_{n}^{\alpha, \beta} \mathscr{K}_{N} v\right\|_{w^{\alpha, \beta}} \leq \xi_{2} \xi_{3}\|v\|_{w^{\alpha, \beta}} \log ^{-1} n .
$$

Again using the same fact that $\lim _{n \rightarrow \infty} \log ^{-1} n=0$, there exists a positive integer $n_{2}$ such that for $n \geq n_{2}$,

$$
\left\|\mathscr{L}_{n}^{\alpha, \beta} \mathscr{K} v-\mathscr{L}_{n}^{\alpha, \beta} \mathscr{K}_{N} v\right\|_{w^{\alpha, \beta}} \leq \frac{\varrho}{4}\|v\|_{w^{\alpha, \beta}} .
$$

When $n \geq n_{0}:=\max \left\{n_{1}, n_{2}\right\}$, these three estimates (108), (112), and (116) yield that

$$
\left\|\left(\mathscr{I}+\mathscr{L}_{n}^{\alpha, \beta} \mathscr{K}_{N}\right) v\right\|_{w^{\alpha, \beta}} \geq \frac{\varrho}{2}\|w\|_{w^{\alpha, \beta}},
$$

which infers our result.

This above result shows that (20) has a unique solution $\widetilde{u}_{n}$ in the space $L_{w^{\alpha, \beta}}^{2}(I)$. Next result considers the approximate order of the solution $\widetilde{u}_{n}$.

Theorem 11. Suppose that the kernel function $k \in C^{m, m}\left(I^{2}\right)$, $f \in C^{m}(I)$, and $-1 / 2<\alpha, \beta<1, \alpha+\beta \leq 1$. If one chooses $N$ as in (109), then there exist a positive constant $\theta$ and a positive integer $n_{0}$ such that, for $n \geq n_{0}$,

$$
\left\|u-\widetilde{u}_{n}\right\|_{w^{\alpha, \beta}} \leq \theta\left(\sum_{i \in \mathbb{Z}_{m}}\left\|\mathscr{D}_{x}^{i} u\right\|_{w^{\alpha+i, \beta+i}}\right) n^{-m} .
$$


TABLE 1: The numerical result based on the collocation nodes $x_{i}^{0,0}, i \in \mathbb{Z}_{n}$.

\begin{tabular}{|c|c|c|c|c|c|c|}
\hline$n$ & 4 & 6 & 8 & 10 & 12 & 14 \\
\hline$\left\|u-\widetilde{u}_{n}\right\|_{\infty}$ & $1.40 e-2$ & $3.16 e-4$ & $4.28 e-6$ & $3.83 e-8$ & $2.60 e-10$ & $1.20 e-12$ \\
\hline$\left\|u-\widetilde{u}_{n}\right\|_{w^{0,0}}$ & $1.85 e-2$ & $4.10 e-4$ & $5.48 e-6$ & $4.87 e-8$ & $3.07 e-10$ & $1.45 e-12$ \\
\hline
\end{tabular}

TABLE 2: The numerical result based on the collocation nodes $x_{i}^{1 / 4,1 / 3}, i \in \mathbb{Z}_{n}$.

\begin{tabular}{lcccccc}
\hline$n$ & 4 & 6 & 8 & 10 & 12 & 14 \\
\hline$\left\|u-\widetilde{u}_{n}\right\|_{\infty}$ & $1.16 e-2$ & $2.61 e-4$ & $3.54 e-6$ & $4.16 e-8$ & $2.14 e-10$ & $9.92 e-13$ \\
$\left\|u-\widetilde{u}_{n}\right\|_{w^{1 / 4,1 / 3}}$ & $1.52 e-2$ & $3.38 e-4$ & $4.52 e-6$ & $4.01 e-8$ & $2.53 e-10$ & $1.19 e-12$ \\
\hline
\end{tabular}

Proof. The proof of Theorem 11 is similar as that of Theorem 7. It follows from Theorem 7 that $u \in C^{m}(I)$, which implies that $u \in H_{w^{\alpha, \beta}}^{m}(I)$. By using the triangle inequality,

$$
\left\|u-\widetilde{u}_{n}\right\|_{w^{\alpha, \beta}} \leq\left\|u-\mathscr{L}_{n}^{\alpha, \beta} u\right\|_{w^{\alpha, \beta}}+\left\|\mathscr{L}_{n}^{\alpha, \beta} u-\widetilde{u}_{n}\right\|_{w^{\alpha, \beta}} .
$$

Upon the estimation in (23) with $i:=0, r:=m$ and $v:=u$, we only need to estimate $\left\|\mathscr{L}_{n}^{\alpha, \beta} u-\widetilde{u}_{n}\right\|_{w^{\alpha, \beta}}$. Employing the result (78), (106), and Theorem 7, there exist a positive constant $\xi_{1}$ and a positive integer $n_{0}$ such that $n \geq n_{0}$,

$$
\left\|\tilde{u}_{n}-\mathscr{L}_{n}^{\alpha, \beta} u\right\|_{w^{\alpha, \beta}} \leq \xi_{1}\left\|\mathscr{K} u-\mathscr{K}_{N} \mathscr{L}_{n}^{\alpha, \beta} u\right\|_{\infty}
$$

To obtain the estimation of the right hand side of (120), we let

$$
\begin{gathered}
I_{1}:=\left\|\mathscr{K} u-\mathscr{K} \mathscr{L}_{n}^{\alpha, \beta} u\right\|_{\infty}, \\
I_{2}:=\left\|\mathscr{K} \mathscr{L}_{n}^{\alpha, \beta} u-\mathscr{K}_{N} \mathscr{L}_{n}^{\alpha, \beta} u\right\|_{\infty} .
\end{gathered}
$$

Clearly,

$$
\left\|\mathscr{K} u-\mathscr{K}_{N} \mathscr{L}_{n}^{\alpha, \beta} u\right\|_{\infty} \leq I_{1}+I_{2} .
$$

Upon the estimation (84), we only require to estimate $I_{2}$. In fact, a combination of (87) and (109) yields that there exists a positive constant $\xi_{2}$ :

$$
I_{2} \leq \xi_{2}\left(\sum_{i \in \mathbb{Z}_{m}}\left\|\mathscr{D}_{x}^{i} u\right\|_{w^{\alpha+i, \beta+i}}\right) n^{-m} \log ^{-1} n
$$

A combination of (84) and (120)-(123) yields the desired result.

Theorem 11 illustrates that the proposed method preserves the optimal order of convergence.

\section{One Numerical Example}

In this section, we are going to present one numerical example to demonstrate the efficiency of the spectral Jacobicollocation method for solving (3). In each example, we use two spectral collocation approaches associated with the weight function $w^{0,0}$ and $w^{1 / 4,1 / 3}$, respectively. Here, we compute the Gauss-Jacobi quadrature rule nodes and weights by Theorems 3.4 and 3.6 discussed in [19]. All computer programs are compiled by Matlab language.
Example. Consider the second kind Volterra integral equation (1) with

$$
k(x, t)=e^{x t}, \quad f(x)=e^{2 x}+\frac{e^{x(x+2)}-e^{-(x+2)}}{x+2} .
$$

The corresponding exact solution is given by $u(x)=e^{2 x}$. As expected, the errors show an exponential decay, since in this semilog representation the error variations are essentially linear versus the degrees of the polynomial.

From the theoretical results we observe that the numerical errors should decay with an exponential rate, and we also find that the errors show an exponential decay (Tables 1 and 2).

\section{Conflict of Interests}

The authors declare that there is no conflict of interests regarding the publication of this paper.

\section{Acknowledgments}

Hongfeng Guo is supported by Natural Science Foundation of China Grant 11026108, Science and Technology Development Project of Shandong Province Grant 2012GGB01241, and Postgraduate Education Innovation Project of Shandong University of Finance and Economics Grant SCY1308. Haotao Cai is supported by Natural Science Foundation of Shandong Province Grant ZR2010AQ001. Xin Zhang is supported by Natural Science Foundation of Shandong Province Grant ZR2010AQ012.

\section{References}

[1] H. Brunner, Collocation Methods for Volterra Integral and Related Functional Equations Methods, Cambridge University Press, Cambridge, UK, 2004.

[2] A. H. Bhrawy, "A Jacobi-Gauss-Lobatto collocation method for solving generalized Fitzhugh-Nagumo equation with timedependent coefficients," Applied Mathematics and Computation, vol. 222, pp. 255-264, 2013.

[3] H. Majidian, "Composite quadrature rules for a class of weakly singular Volterra integral equations with noncompact kernels," Applied Numerical Mathematics, vol. 83, pp. 1-11, 2014.

[4] Z. Chen and W. Jiang, "The exact solution of a class of Volterra integral equation with weakly singular kernel," Applied 
Mathematics and Computation, vol. 217, no. 18, pp. 7515-7519, 2011.

[5] Y. Chen and T. Tang, "Spectral methods for weakly singular Volterra integral equations with smooth solutions," Journal of Computational and Applied Mathematics, vol. 233, no. 4, pp. 938-950, 2009.

[6] S. Pishbin, F. Ghoreishi, and M. Hadizadeh, "The semi-explicit Volterra integral algebraic equations with weakly singular kernels: the numerical treatments," Journal of Computational and Applied Mathematics, vol. 245, pp. 121-132, 2013.

[7] G. Zakeri and M. Navab, "Sinc collocation approximation of non-smooth solution of a nonlinear weakly singular Volterra integral equation," Journal of Computational Physics, vol. 229, no. 18, pp. 6548-6557, 2010.

[8] Y. Jiang and J. Ma, "Spectral collocation methods for Volterraintegro differential equations with noncompact kernels," Journal of Computational and Applied Mathematics, vol. 244, pp. 115-124, 2013.

[9] G. N. Elnagar and M. Kazemi, "Chebyshev spectral solution of nonlinear Volterra-Hammerstein integral equations," Journal of Computational and Applied Mathematics, vol. 76, no. 1-2, pp. 147-158, 1996.

[10] H. Fujiwara, "High-accurate numerical method for integral equations of the first kind under multipleprecision arithmetic," Preprint, RIMS, Kyoto University, 2006.

[11] T. Tang, X. Xu, and J. Cheng, "On spectral methods for Volterra integral equations and the convergence analysis," Journal of Computational Mathematics, vol. 26, no. 6, pp. 825-837, 2008.

[12] Y. Chen and T. Tang, "Convergence analysis of the Jacobi spectral-collocation methods for Volterra integral equations with a weakly singular kernel," Mathematics of Computation, vol. 79, no. 269, pp. 147-167, 2010.

[13] Z. Xie, X. Li, and T. Tang, "Convergence analysis of spectral Galerkin methods for Volterra type integral equations," Journal of Scientific Computing, vol. 53, no. 2, pp. 414-434, 2012.

[14] Y. Wei and Y. Chen, "Legendre spectral collocation methods for pantograph Volterra delay-integro-differential equations," Journal of Scientific Computing, vol. 53, no. 3, pp. 672-688, 2012.

[15] H. T. Cai, "A Jacobi-collocation method for solving second kind Fredholm integral equations with weakly singular kernels," Science China: Mathematics, 2014.

[16] M. R. Hajmohammadi, S. S. Nourazar, and A. H. Manesh, "Semi-analytical treatments of conjugate heat transfer," Journal of Mechanical Engineering Science, vol. 227, no. 3, pp. 492-503, 2012.

[17] M. R. Hajmohammadi and S. S. Nourazar, "On the solution of characteristic value problems arising in linear stability analysis; semi analytical approach," Applied Mathematics and Computation, vol. 239, pp. 126-132, 2014.

[18] M. R. Hajmohammadi, R. Mohammad, and S. S. Nourazar, "Conjugate forced convection heat transfer from a heated flat plate of finite thickness and temperature-dependent thermal conductivity," Heat Transfer Engine, vol. 35, pp. 863-874, 2014.

[19] J. Shen, T. Tang, and L. Wang, Spectral Methods: Algorithms, Analysis and Applications, vol. 41 of Springer Series in Computational Mathematics, Springer, New York, NY, USA, 2011.

[20] G. Mastroianni and M. G. Russo, "Lagrange interpolation in weighted Besov spaces," Constructive Approximation, vol. 15, no. 2, pp. 257-289, 1999.

[21] D. L. Ragozin, "Polynomial approximation on compact manifolds and homogeneous spaces," Transactions of the American Mathematical Society, vol. 150, pp. 41-53, 1970.
[22] D. L. Ragozin, "Constructive polynomial approximation on spheres and projective spaces," Transactions of the American Mathematical Society, vol. 162, pp. 157-170, 1971.

[23] R. Kress, Linear Integral Equations, Springer, New York, NY, USA, 1st edition, 1989. 


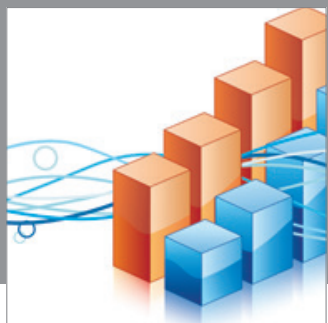

Advances in

Operations Research

mansans

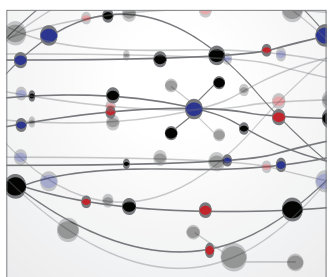

The Scientific World Journal
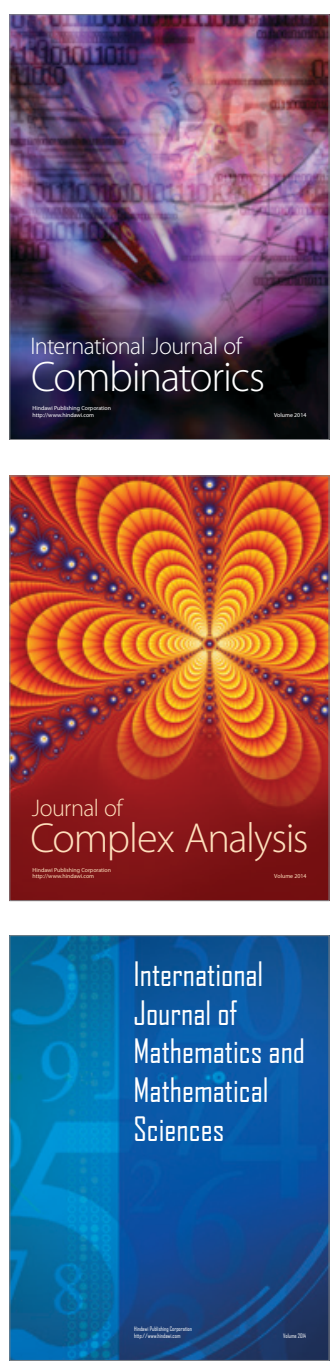
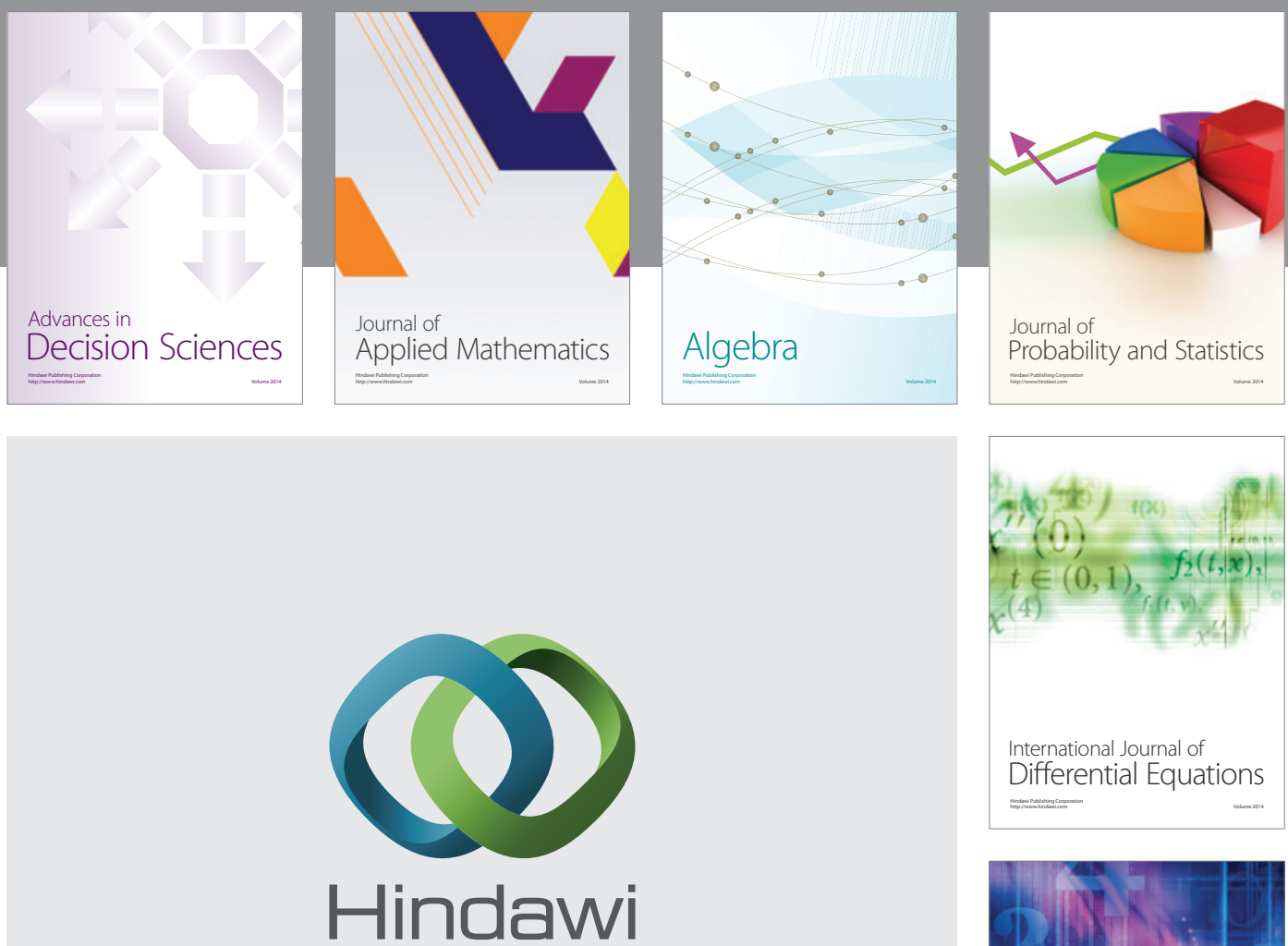

Submit your manuscripts at http://www.hindawi.com
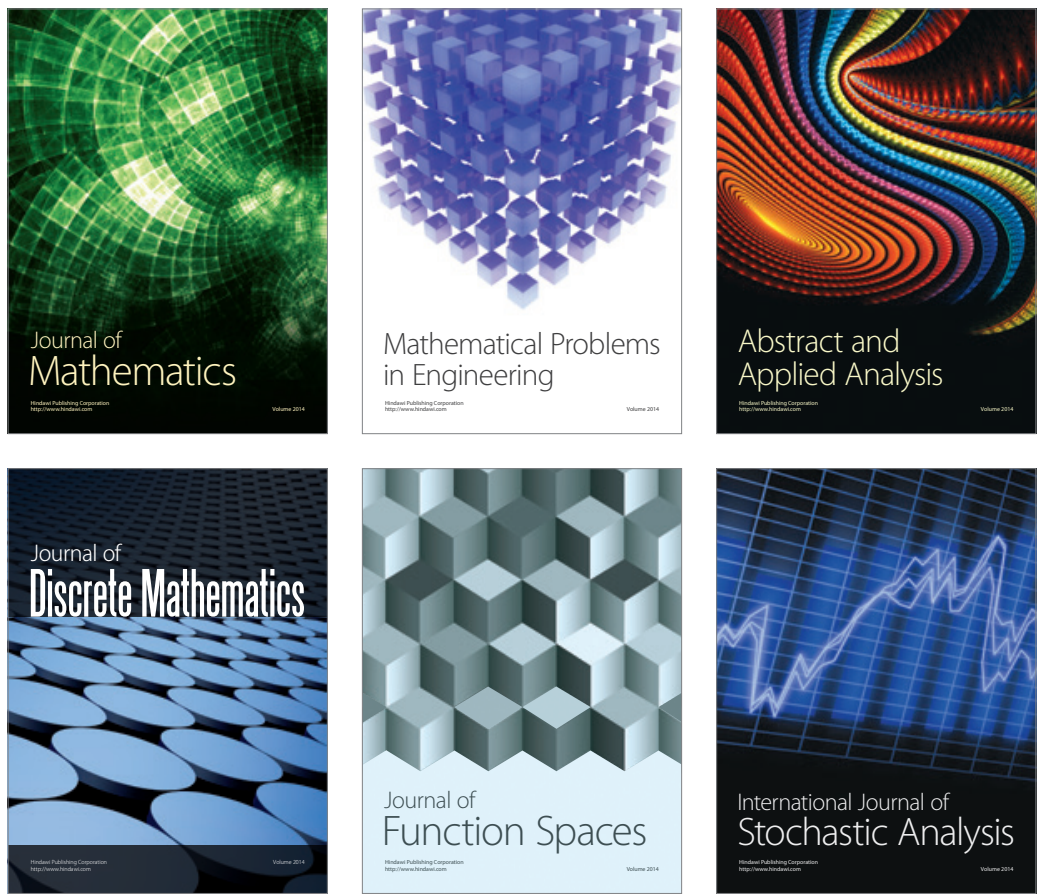

Journal of

Function Spaces

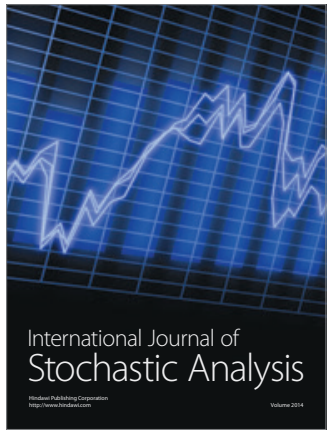

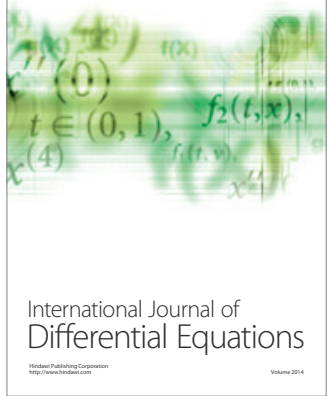
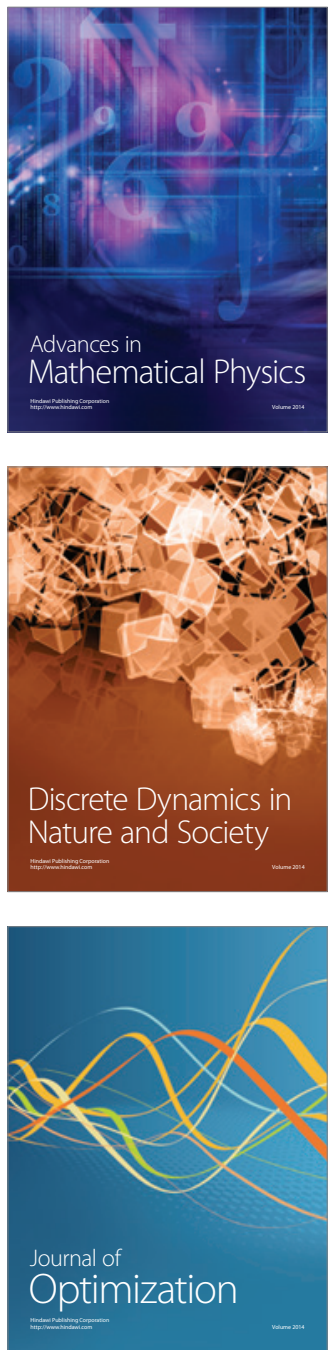